\title{
Program Parallelization Using Synchronized Pipelining
}

\author{
Leonardo Scandolo ${ }^{1}$, César Kunz ${ }^{1}$, and Manuel Hermenegildo ${ }^{1,2}$ \\ 1 IMDEA Software \\ 2 Technical U. of Madrid, Spain \\ Firstname.Lastname@imdea.org
}

\begin{abstract}
While there are well-understood methods for detecting loops whose iterations are independent and parallelizing them, there are comparatively fewer proposals that support parallel execution of a sequence of loops or nested loops in the case where such loops have dependencies among them. This paper introduces a refined notion of independence, called eventual independence, that in its simplest form considers two loops, say loop ${ }_{1}$ and loop $_{2}$, and captures the idea that for every $i$ there exists $k$ such that the $i+1$-th iteration of loop $_{2}$ is independent from the $j$-th iteration of loop $_{1}$, for all $j \geq k$. Eventual independence provides the foundation of a semantics-preserving program transformation, called synchronized pipelining, that makes execution of consecutive or nested loops parallel, relying on a minimal number of synchronization events to ensure semantics preservation. The practical benefits of synchronized pipelining are demonstrated through experimental results on common algorithms such as sorting and Fourier transforms.
\end{abstract}

\section{Introduction}

Multi-core processors are becoming ubiquitous: most laptops currently on the market contain at least two execution units, whereas servers commonly use eight or more cores. Since the number of on-chip cores is expected to double with each processor generation, there is a pressing challenge to develop programming methodologies which exploit the power of multi-core processors without compromising correctness and reliability. One prominent approach is to let programmers write sequential programs and to build compilers that parallelize these programs automatically.

Most parallelization techniques rely on some notion of independence, which ensures that certain fragments of the program only access distinct regions of memory, and thus execution of one such code fragment has no effect on the execution of the others. For example, code fragments written in a simple imperative language are guaranteed to be independent if their reads and writes are disjoint, in which case their sequential composition can be parallelized without modifying the overall semantics of the program. More refined notions of independence include the classical notions of absence of flow dependence, anti-dependence, or output dependence [7]. 
Well-understood methods exist for detecting loops whose iterations are independent (i.e., they do not contain loop-carried dependencies) and parallelizing them. These techniques have been used to achieve automated/correct parallelization of a number of algorithms for scientific computing such as, e.g., image processing, data mining, DNA analysis, or cosmological simulation. However, these parallelization methods do not provide significant speedups for other algorithms which contain sequences or nesting of loops whose iterations are partially dependent and/or irregular. Examples of such loops appear, for example, in sorting algorithms or Fourier transformations. On the other hand, such algorithms can be parallelized efficiently by the technique that we propose, synchronized pipelining, which allows loops with dependencies to be executed in parallel by making sparse use of synchronization events to ensure that the ahead-of-time execution of loop iterations does not alter the original semantics.

Our proposal is illustrated in Section 2 with a mergesort algorithm. As a warm-up to Section 2, let us first consider synchronized pipelining in its simplest form, when it deals with two consecutive loops manipulating an array structure:

while $b_{1}$ do $c_{1}$; while $b_{2}$ do $c_{2}$

For simplicity, assume that the data dependence between $c_{1}$ and $c_{2}$ is restricted to the contents of the array structure. The aim is to return code that may start the execution of some iterations of $c_{2}$ before completion of the loop with body $c_{1}$. To justify such a transformation, we rely on eventual independence, a generalization of independence which accounts for the possibility of executing the $m+1$-th iteration of a loop ahead of time. Informally, $c_{2}$ is eventually independent from $c_{1}$ iff for every $n_{2}$, there exists $n_{1}$ such that after $n_{1}$ iterations of $c_{1}$ and $n_{2}$ iterations of $c_{2}, c_{1}$ and $c_{2}$ are independent. Once eventual independence between the two loops is established, it is possible to define a semantics-preserving transformation that outputs a program:

while $b_{1}$ do $c_{1}^{\prime} \|$ while $b_{2}$ do $c_{2}^{\prime}$

where $c_{1}^{\prime}$ is obtained from $c_{1}$ by adding event announcements to indicate that part of the computation of $c_{2}$ can be performed, and $c_{2}^{\prime}$ is obtained from $c_{2}$ by inserting blocking statements that control the gradual and early computation of $c_{2}$; in both cases, the transformation of $c_{i}$ into $c_{i}^{\prime}$ is guided by the eventual independence relation.

In the course of the paper, we develop the notions of eventual independence and synchronized pipelining, starting from the simple case discussed above and then dealing with sequences of loops and nested loops. In addition, we illustrate the benefits of our approach, drawing experimental results from common cases such as the above mentioned sorting algorithms and Fourier transforms. We also outline the necessary procedures and tools to automatically generate this transformation for the case in which we deal with simple data structures (arrays), and outline future lines of research to extend this approach to more general problems. In summary, the main contributions of this paper are the formal definition of eventual independence (Section 4), eventual independence 


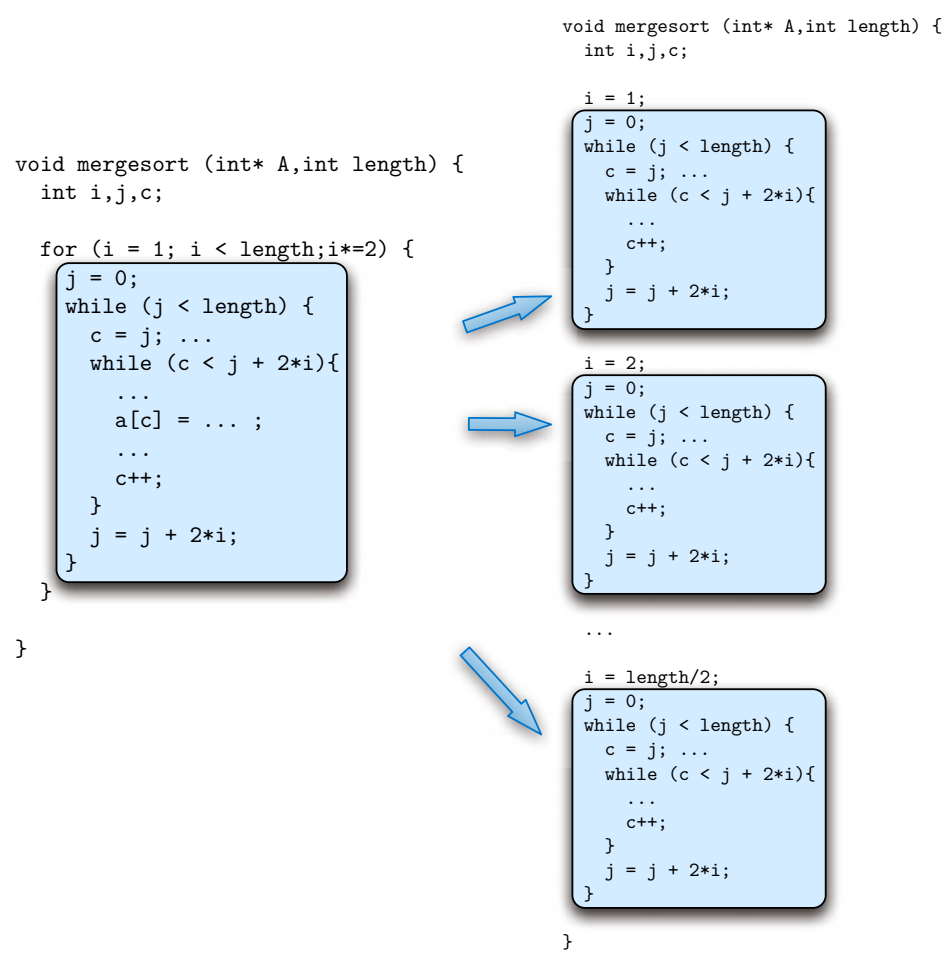

Fig. 1. Iterative mergesort algorithm

criteria for the particular case of array manipulating loops, and an experimental evaluation of the benefits of synchronized pipelining (Section 6). Although many of the concepts and results of the paper only make minimal assumptions on the programming language, we carry our development in the setting of a parallel imperative language with events, introduced in Section 3 .

\section{Motivating Example: Mergesort}

Figure 1 presents the structure of an iterative mergesort algorithm. After unrolling some of the for loop iterations from the fragment shown on the right of the figure, we have a sequence of iterations of the inner loop while $(j<$ length $\{\ldots\}$ accessing and modifying the array intervals $[0,1],[2,3], . .$, length1 , length $]$ in the first iteration, the intervals $[0,3],[4,7], \ldots,[$ length -3 , length] in the second iteration, and so on until the last iteration in which the intervals $[0$, length $/ 2]$ and $[$ length $/ 2+1$, length $]$ are accessed.

One can clearly see that the first and second unrolled iteration cannot be executed in parallel (without changes) since they read and/or modify overlapping regions of the array. However, after partial completion of the first iteration, the 


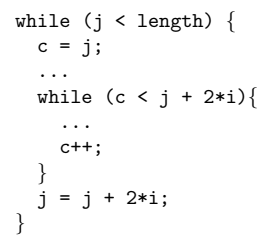

(a) Original

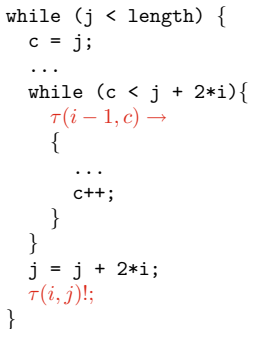

(b) Pipelined

Fig. 2. Illustrative example of pipelined code

second iteration can advance without waiting for the first iteration to finish. For instance, the second iteration can safely start processing the array interval $[0,3]$, right after the first iteration has finished processing the array intervals $[0,1]$ and $[2,3]$. The parallelization technique we propose allows the second loop iteration to gradually progress in parallel with the first one (and successive ones), introducing synchronization primitives in order to preserve the original semantics. To this end, we rely on a heuristic oracle $\Omega$, defined in terms of the number of steps already executed by the first and second loop, that determines at which point of the first loop it is safe to enable a partial execution of the second one.

Figure 2 gives a brief but illustrative scheme of how the code in Figure 1 is to be annotated with parallelization primitives. In this case we use $\tau$ to denote such device, using a question mark to signify a wait event on a certain subscript (or set of subscripts) that the current loop is waiting to use, and an exclamation mark to denote a signaling event which allows other threads to continue execution.

\section{$3 \quad$ Setting}

The target language for synchronized pipelining is a simple imperative language with arrays, extended with parallel composition and synchronization primitives.

The extension includes an empty statement nil, a standard parallel composition $\|$, and event-based synchronization primitives. We assume given a set of events $\mathcal{S}$ used for synchronization. Let $\tau \in \mathcal{S}$ and $S \subseteq \mathcal{S}$ represent a synchronization event and a synchronization event set, respectively. The statement $S$ ! is a non-blocking announcement of the events in $S$, whereas the statement $\tau \rightarrow c$ waits for the event $\tau$ to be announced before proceeding with the execution of $c$.

Let Stmt be the set of program statements, $\Sigma$ be the set of mappings from program variables to integer values, and $\mathcal{S}^{\star}$ be the powerset of $\mathcal{S}$. The program semantics is given by a transition relation between configurations, where a configuration is either an exceptional configuration abort, resulting, e.g., from and array-out-of-bound access, or a normal configuration, i.e., an element of Stmt $\times \Sigma \times \mathcal{S}^{\star}$. Formally, the semantics is given by a small-step relation: $\rightsquigarrow \subseteq$ $\left(\right.$ Stmt $\left.\times \Sigma \times \mathcal{S}^{\star}\right) \times\left(\left(\right.\right.$ Stmt $\left.\times \Sigma \times \mathcal{S}^{\star}\right)+\{$ abort $\left.\}\right)$. 


$$
\begin{aligned}
& \overline{\langle S !, \sigma, \epsilon\rangle \rightsquigarrow\langle\text { nil, } \sigma, \epsilon \cup S\rangle} \\
& \frac{c \equiv d \quad\langle d, \sigma, \epsilon\rangle \rightsquigarrow\left\langle d^{\prime}, \sigma^{\prime}, \epsilon^{\prime}\right\rangle \quad d^{\prime} \equiv c^{\prime}}{\langle c, \sigma, \epsilon\rangle \rightsquigarrow\left\langle c^{\prime}, \sigma^{\prime}, \epsilon^{\prime}\right\rangle} \quad \frac{c \equiv d \quad\langle d, \sigma, \epsilon\rangle \rightsquigarrow \text { abort }}{\langle c, \sigma, \epsilon\rangle \rightsquigarrow \text { abort }} \\
& \frac{\langle c, \sigma, \epsilon\rangle \rightsquigarrow\left\langle c^{\prime}, \sigma^{\prime}, \epsilon^{\prime}\right\rangle}{\langle c \| d, \sigma, \epsilon\rangle \rightsquigarrow\left\langle c^{\prime} \| d, \sigma^{\prime}, \epsilon^{\prime}\right\rangle} \quad \frac{\langle c, \sigma, \epsilon\rangle \rightsquigarrow \text { abort }}{\langle c \| d, \sigma, \epsilon\rangle \rightsquigarrow \text { abort }} \\
& i \| \text { nil } \equiv i \quad i\|j \equiv j\| i \quad i\|(j \| k) \equiv(i \| j)\| k
\end{aligned}
$$

Fig. 3. Operational semantics (excerpts)

The transition rules for synchronization and parallel execution are given in Figure 3, together with the definition of the congruence relation $\equiv \subseteq S t m t \times S t m t$; all other rules are standard. Note that event announcement is asynchronous and that event identifiers are never removed from $\epsilon$. Thus, once an event has been announced, and until the end of the program execution, every process waiting for that event is ready to proceed.

Example 1. Consider for example the statement $(\mathrm{x}:=5 ; \tau !) \| \tau \rightarrow \mathrm{x}:=1$. Starting from a state where $\tau$ has not been announced, the execution terminates with the variable $x$ holding the value 1 , since $\mathrm{x}:=1$ cannot proceed before the event $\tau$ has been announced.

As usual, we can derive from the small-step semantics an evaluation semantics $\Downarrow \subseteq\left(\right.$ Stmt $\left.\times \Sigma \times \mathcal{S}^{\star}\right) \times(\Sigma+$ abort $)$, by setting:

$$
\begin{array}{lcc}
\langle c, \sigma, \epsilon\rangle \Downarrow \sigma^{\prime} & \text { iff } & \exists \epsilon^{\prime} .\langle c, \sigma, \epsilon\rangle \rightsquigarrow^{\star}\left\langle\text { nil, } \sigma^{\prime}, \epsilon^{\prime}\right\rangle \\
\langle c, \sigma, \epsilon\rangle \Downarrow \text { abort } & \text { iff } & \langle c, \sigma, \epsilon\rangle \rightsquigarrow^{\star} \text { abort }
\end{array}
$$

where $\rightsquigarrow^{\star}$ denotes the reflexive and transitive closure of $\rightsquigarrow$. In turn, the evaluation semantics can be used to define a notion of semantic equivalence.

Definition 1 (Semantic Equivalence). Let $c_{1}, c_{2} \in$ Stmt be two statements, $\sigma \in \Sigma$ be a state and $\epsilon \subseteq \mathcal{S}$ be a set of synchronization events. We say that $c_{2}$ simulates $c_{1}$ w.r.t. $\sigma$ and $\epsilon$, written $\llbracket c_{1} \rrbracket \leq_{(\sigma, \epsilon)} \llbracket c_{2} \rrbracket$, iff for every $\sigma^{\prime} \in(\Sigma+$ abort), we have $\left\langle c_{1}, \sigma, \epsilon\right\rangle \Downarrow \sigma^{\prime} \Rightarrow\left\langle c_{2}, \sigma, \epsilon\right\rangle \Downarrow \sigma^{\prime}$. We say that $c_{1}$ and $c_{2}$ are semantically equivalent w.r.t. $\sigma$ and $\epsilon$, written $\llbracket c_{1} \rrbracket \equiv_{(\sigma, \epsilon)} \llbracket c_{2} \rrbracket$, iff $\llbracket c_{1} \rrbracket \leq_{(\sigma, \epsilon)} \llbracket c_{2} \rrbracket$ and $\llbracket c_{2} \rrbracket \leq_{(\sigma, \epsilon)} \llbracket c_{1} \rrbracket$.

\section{Eventual Independence}

The purpose of this section is to introduce the notion of eventual independence, and to discuss how eventual independence relations may be inferred. For the sake of completeness, we start by recalling the semantic notion of independence between two statements. 
Definition 2 (Independent Statements). Two statements $c_{1}, c_{2} \in$ Stmt are independent iff $\llbracket c_{1} ; c_{2} \rrbracket \equiv \llbracket c_{1} \| c_{2} \rrbracket$.

Eventual independence aims to capture a relation between iterations of two loop bodies $c_{1}$ and $c_{2}$, and thus would be naturally formalized as a relation between natural numbers. For the clarity of the technical development, it is however preferable to view eventual independence as a relation between natural numbers and events, and assume given a function $\lambda: \mathbb{N} \rightarrow \mathcal{S}$ that assigns to each natural number $m$ of loop ${ }_{2}$ the event $\lambda(m)$ that will release the $m$-th iteration of loop ${ }_{2}$.

Definition 3 (Eventual Independence Relation). Statements $c_{1}, c_{2} \in$ Stmt are eventually independent w.r.t. a relation $\Omega \subseteq \mathbb{N} \times \mathcal{S}$ iff for all $m, n \in \mathbb{N}, \epsilon \subseteq \mathcal{S}$ s.t. $(n, \lambda(m)) \in \Omega, \sigma \in \Sigma$ and no synchronization variables in $\epsilon$ appear in $c_{1}$ or $c_{2}$ :

$$
\llbracket c_{1}^{n} ; c_{2}^{m-1} ; c_{1}^{k} ; c_{2} \rrbracket \equiv{ }_{(\sigma, \epsilon)} \llbracket c_{1}^{n} ; c_{2}^{m-1} ;\left(c_{1}^{k} \| c_{2}\right) \rrbracket
$$

for all $k \in \mathbb{N}$. The expression $c^{i}$ stands for the sequential composition of $i$ instances of the statement c. Given $\Omega$ and $n \in \mathbb{N}$, we let $\omega(n)=\{s \mid(n, s) \in \Omega\}$.

Example 2. Consider the following program:

while $b_{i}$ do $\{a[i]:=a[i]+1 ; i:=i * 2\} ;$ while $b_{j}$ do $\{a[j]:=a[j]+1 ; j:=j+1\}$

The two loop statements are not necessarily independent, but one can define an eventual independence relation over the loop bodies in order to parallelize their iterations. In this case, the loop statements are eventually independent with respect to a relation $\Omega$, if $(n, \lambda(m)) \in \Omega$ implies $i^{\star} * 2^{n}<j^{\star}+m$, where $i^{\star}$ is the initial value of variable $i$ and $j^{\star}$ is the initial value of variable $j$.

In practice, when considering sequential code, it is sufficient to state the semantics equivalence in terms of the event set $\epsilon=\emptyset$. From the definition of eventual independence, if $\lambda(m)=s$, then the $m^{\text {th }}$ execution of $c_{2}$ shall wait for the event $s$ to execute. Assuming $(n, s) \in \Omega$ then it is safe to signal the event $s$ after executing $n$ times the statement $c_{1}$, allowing the $m^{\text {th }}$ execution of statement $c_{2}$ to take place. Indeed, by definition of $\Omega$, it follows from $(n, s) \in \Omega$ that after $n$ iterations of $c_{1}$, any and all subsequent executions of $c_{1}$ do not modify a piece of memory on which the $m^{\text {th }}$ iteration of $c_{2}$ depends.

The main reason for defining the $\Omega$ relation is to link the iterations of the loop bodies that are safe to execute in parallel. If we take $m=1$ in the definition, then we see that $n$ is simply the number of iterations of $c_{1}$ that we need to execute before we can execute the first iteration of $c_{2}$ (in parallel with the remaining iterations of the first loop) without altering the semantics of the original program. Higher values of $m$ are in relation through $\Omega$ with the values $n$ after which it is safe to execute the $m^{t h}$ iteration of the second loop, provided that the $m-1$ previous iterations where executed following the guidelines that $\Omega$ defines. This is the basis for the transformation we are aiming at and it is formalized in the next section.

The set $\omega(n)$, which is defined in terms of $\Omega$, is the set of all the events that are safe to announce after $n$ executions of the statement $c_{1}$. Since the purpose 
of this definition is to have a construct that will allow us to denote the set of events the first loop can safely announce after each iteration has ended, we will mainly use $\omega$ when defining our transformation.

\subsection{Inferring Eventual Independence}

The eventual independence relation $\Omega$ and the function $\lambda$ are essential ingredients of synchronized pipelining, as they will be used to guide the insertion of synchronization statements in the original program. Therefore, it is important to be able to infer $\Omega$ and $\lambda$ for a large class of code fragments. We have been able to infer this data efficiently for the algorithms under consideration, that manipulate array structures of significant size. Consider the case in which both $c_{1}$ and $c_{2}$ read and modify data from a single array a, iterating over the induction variables $h_{1}$ and $h_{2}$ respectively. By simple code inspection, one can easily collect the sets of syntactic expressions $\boldsymbol{e}_{1}$ and $\boldsymbol{e}_{2}$ used to read or update the array a inside the loop body. These array accesses are not always expressed in terms of the induction variables $h_{1}$ and $h_{2}$. However, in general, we have found that they are expressed in terms of induction variables $h_{1}^{\prime}$ and $h_{2}^{\prime}$ derived from $h_{1}$ and $h_{2}$. In those cases, induction variable analysis 4] allows one to rewrite the derived induction variables $h_{1}^{\prime}$ and $h_{2}^{\prime}$ in terms of the induction variables $h_{1}$ and $h_{2}$, i.e. $h_{1}^{\prime}=f_{1}\left(h_{1}\right)$ and $h_{2}^{\prime}=f_{2}\left(h_{2}\right)$ for some function expressions $f_{1}$ and $f_{2}$.

Most frequently, when $h_{i}^{\prime}$ is an induction variable derived from $h_{i}$, then $f_{i}$ is a linear function on $h_{i}$. More complex cases may arise, for instance when $f_{i}$ is defined as a polynomial or geometric function on $h_{i}$. In those cases, the expressions $\boldsymbol{e}_{1}\left(h_{1}^{\prime}\right)$ and $\boldsymbol{e}_{2}\left(h_{1}^{\prime}\right)$ are easily rewritten in terms of the inductive variables, i.e., as $\boldsymbol{e}_{1}\left(f_{1}\left(h_{1}\right)\right)$ and $\boldsymbol{e}_{2}\left(f_{2}\left(h_{2}\right)\right)$. By static interval analysis, we can approximate the regions of data that are read and modified by $c_{1}$ and $c_{2}$, in terms of the induction variables $h_{1}$ and $h_{2}$, and the expressions $\boldsymbol{e}_{1}\left(f_{1}\left(h_{1}\right)\right)$ and $e_{2}\left(f_{2}\left(h_{2}\right)\right)$.

Assume $\left[l_{1}^{r w}, u_{1}^{r w}\right]$ represents the interval of the array a that is written or read by $c_{1}$, where $l_{1}^{r w}, u_{1}^{r w}$ are integer expressions that depend on $h_{1}$ (and similarly with $\left.c_{2}\right)$. Since $\boldsymbol{e}\left(f_{1}\left(h_{1}\right)\right)$ and $\boldsymbol{e}\left(f_{2}\left(h_{2}\right)\right)$ are linear (or polynomial) functions on $h_{1}$ and $h_{2}$, one can determine whether they are monotonic (or determine the points from which they are monotonic). If the $l$ and $u$ expressions are increasing as the $h$ variables grow (the decreasing case is symmetrical) one can propose an eventual independence relation $\Omega$. For instance when $l_{1}^{r w}$ and $u_{2}^{r w}$ are increasing functions, we determine the pairs $(a, b)$ of values for $h_{1}$ and $h_{2}$ such that $u_{2}^{r w}<l_{1}^{r w}$, and then, since the $b^{\text {th }}$ iteration of $c_{2}$ is independent of the $a^{\text {th }}$ iteration of $c_{1}$, we can have $(a, \lambda(b)) \in \Omega$.

Example 3. We show in this paragraph how to determine an eventual independence relation for this simple pair of loop statements

$$
\text { while } b_{1} \text { do } c_{1} \text {; while } b_{2} \text { do } c_{2}
$$


where $c_{1}$ and $c_{2}$ are defined as

$$
\begin{aligned}
& c_{1} \doteq \mathrm{a}[\mathrm{x}]:=1 ; \mathrm{x}:=\mathrm{x}+1 \\
& c_{2} \doteq \mathrm{y}:=\mathrm{y}+\mathrm{a}[\mathrm{z}] ; \mathrm{z}:=\mathrm{z}+1
\end{aligned}
$$

First of all, notice that statements $c_{1}$ and $c_{2}$ access the array a, so they are not independent. By examining statements $c_{1}$ and $c_{2}$, it is immediate that the indexes of the array accesses are monotonically increasing and the relation between the initial values of program variables (denoted $x^{\star}$ for a variable $x$ ) define the eventual independence relation. In this case, a simple induction variable analysis will define $\boldsymbol{e}_{1}$ and $\boldsymbol{e}_{2}$, and thus $l_{1}^{r w}, l_{2}^{r w}, u_{1}^{r w}$ and $u_{2}^{r w}$, as a linear function of the induction variables: $l_{1}^{r w}\left(h_{1}\right)=u_{1}^{r w}\left(h_{1}\right)=h_{1}+x^{\star}$ and $l_{2}^{r w}\left(h_{2}\right)=u_{2}^{r w}\left(h_{2}\right)=h_{2}+z^{\star}$. Thus, the procedure's requirements translate into: $h_{2}+z^{\star}<h_{1}+x^{\star}$. The argument above allows us to propose an eventual independence relation $\Omega$.

$$
\begin{aligned}
& \left(z^{\star}-x^{\star}+1, \lambda(1)\right) \in \Omega_{c_{1}, c_{2}} \\
& \forall x . x \leq z^{\star}-x^{\star}+1 \Rightarrow(x, \lambda(1)) \notin \Omega_{c_{1}, c_{2}}
\end{aligned}
$$

This $\Omega$ relation formalizes the intuition that $c_{1}$ and $c_{2}$ can be executed in parallel as long as every iteration $k$ of $c_{2}$ executes after the iteration number $z^{\star}-x^{\star}+k$ of $c_{1}$. Furthermore, since the size of the array a $(|\mathrm{a}|)$ is bounded, if $c_{1}$ is executed more than $|\mathrm{a}|-x^{\star}$ times, we end up at an exceptional state abort, in which case any execution of $c_{2}$ is independent. In conclusion, the following relation $\Omega$ determines the eventual independence between $c_{1}$ and $c_{2}$ :

$$
\begin{aligned}
& x+x^{\star} \leq|\mathrm{a}| \wedge y \leq x+z^{\star}-x^{\star}-1 \Rightarrow(x, \lambda(y)) \in \Omega_{c_{1}, c_{2}} \\
& x+x^{\star}>|\mathrm{a}| \Rightarrow(x, \lambda(y)) \in \Omega_{c_{1}, c_{2}}
\end{aligned}
$$

\section{Synchronized Pipelining}

We now define synchronized pipelining, starting from two consecutive loops, and then extending the transformation to sequences of loops and nested loops.

Consider a program $c$ of the form while $b_{1}$ do $c_{1}$; while $b_{2}$ do $c_{2}$, where $c_{1}$ and $c_{2}$ are compound statements that access an array. We assume that the boolean conditions $b_{1}$ and $b_{2}$ are not affected by the execution of $c_{2}$ and $c_{1}$, respectively. Further, we let $h_{1}$ and $h_{2}$ be program counters that determine the number of iterations already performed for the first and second loop respectively. Our aim is to transform the program so that it executes both loops in parallel. To preserve the program semantics, the transformation must insert code that ensures a correct synchronization between the two loops, so the resulting program will be of the form while $b_{1}$ do $c_{1}^{\prime} \|$ while $b_{2}$ do $c_{2}^{\prime}$, where $c_{1}^{\prime}$ is derived from $c_{1}$ by adding event announcements and $c_{2}^{\prime}$ is derived from $c_{2}$ by adding synchronization guards. Both transformations are guided by a relation $\Omega$ of eventual independence and by a function $\lambda$ that are given as input to the transformation. 
Definition 4. The synchronized pipelining of $c$ is statement $\overline{\bar{c}}$ defined as:

$$
\overline{\bar{c}}=\left(\text { while } b_{1} \text { do } c_{1}^{\prime}\right) ; S ! \| \text { while } b_{2} \text { do } c_{2}^{\prime}
$$

where $c_{1}^{\prime}=c_{1} ; \omega\left(h_{1}\right) !, c_{2}^{\prime}=\lambda\left(h_{2}\right) \rightarrow c_{2}$, and $S$ is the set of all events on which statement $c_{2}^{\prime}$ can wait.

Statement $S$ ! is introduced after the execution of $c_{1}^{\prime}$ to ensure that all events are indeed announced, and thus the progress of the original program is preserved. In order to accomplish that, statement $S$ ! simply announces all events, in any order. Since all events in which statement $c_{2}^{\prime}$ is waiting are eventually announced by $S$, statement $c_{2}^{\prime}$ cannot block indefinitely. For the same reason, $c \leq \overline{\bar{c}}$. Notice that the set of events announced by $c_{1}^{\prime}$ and $S$ ! may be redundant. In practice, one can reduce program size and synchronization overhead by statically removing duplicated events. Similarly, $c_{2}$ may be simplified by removing synchronization primitives that wait on the same event. We assume, however, the definition given above for notational simplicity.

The eventual independence condition determined by $\Omega$ is enough to show that the semantics is preserved. That is, every execution state reached by the final program is also reachable by the original one.

Proposition 1 (Semantics Preservation). For every initial state $\sigma \in \Sigma$ and every event set $\epsilon$ disjoint from the fresh synchronization variables introduced by the transformation, we have that $\llbracket c \rrbracket \equiv{ }_{(\sigma, \epsilon)} \llbracket \overline{\bar{c}} \rrbracket$.

\subsection{Extensions}

We first analyze the case of a sequence of loops. Then, we explain how we proceed in the presence of nested loops.

Loop Sequences. Now suppose the original program is of the form:

$$
\text { while } b_{1} \text { do } c_{1} ; \ldots \text {; while } b_{n} \text { do } c_{n}
$$

The idea is to parallelize the whole program by progressively applying the basic transformation to each pair of interfering loops. Therefore, we must provide for all $i, j$ such that $i<j$ an eventual independence relation $\Omega_{i, j}$ and a function $\lambda_{i, j}: \mathbb{N} \rightarrow \mathcal{S}$. By definition of eventual independence, we must have for every $\left(n, \lambda_{i, j}(m)\right) \in \Omega_{i, j}$ and for all state $\sigma$ and event set $\epsilon$ :

$$
\llbracket c_{i}^{n} ; c_{j}^{m-1} ; c_{i}^{k} ; c_{j} \rrbracket \equiv{ }_{(\sigma, \epsilon)} \llbracket c_{i}^{n} ; c_{j}^{m-1} ;\left(c_{i}^{k} \| c_{j}\right) \rrbracket
$$

Since the parallel execution of the $i^{\text {th }}$ loop may interfere not only with its immediately preceding loop, but with every preceding one, we synchronize each pair of non-independent loops. Thus, the $i^{\text {th }}$ loop of the final program becomes:

$$
\text { while } b_{i} \text { do } \bigcup_{1 \leq j<i} \lambda_{i, j}(h) \rightarrow\left(c_{i} ; \bigcup_{i<j \leq n} \omega_{i, j}(h) !\right) ; \forall_{i<j \leq n} S_{i, j} \text { ! }
$$


where $S_{i, j}$ stands for all the synchronization events used to synchronize execution between while $b_{i}$ do $c_{i}$ and while $b_{j}$ do $c_{j}$, for every $i<j$. From the expression above, it may seem that excessive synchronization overhead is introduced. However, the actual number of synchronization primitives depends on the definition of $\lambda$ and $\omega$, and on the removal of duplicated synchronization events.

Nested Loops. We now turn our attention to a different but more common program structure: nested loops. Consider the following program as the target of the parallelization: while $a$ do $\left(c_{1}\right.$; while $b$ do $\left.c ; c_{2}\right)$. In order to be able to apply our transformation we take the following assumptions:

1. We assume that the number of iterations of the outer loop (or an overapproximation) can be computed at runtime. In the rest of this section we let $\beta$ stand for the number of iterations that may be computed at runtime and, for simplicity, we assume that the boolean condition $a$ is of the form $l \leq \beta$, where $l$ is the induction variable of the outer loop, incremented with step 1 from the initial value 1 . In practice, the exact form of $a$ may differ from this assumption, but we assume that it is possible to evaluate the number of iterations at runtime based on the current memory state. Intuitively, if we can determine the exact number of iterations of the outer loop, we can unroll it and parallelize the resulting program by applying the transformation on sequences of loops as explained above. However, assuming that we can statically determine the exact number of iterations is an unnecessary and too strong assumption.

2. We assume also that there is no interference between the scalar variables read and modified in $c_{1}$ and $c$. We can reduce the interference between loop iterations by vectorizing each scalar variable $v$ into an array $\hat{v}$, with the cost of extra memory usage. For every statement $c$ and boolean condition $b$, we denote $\hat{c}[l]$ and $\hat{b}[l]$ the result of vectorizing scalar variables in $c$ and $b$, respectively. The value of the variable $l$ determines which position of the vectorized variables is in use. At the end of the transformed program, a sync operation takes each vectorized variable $\hat{v}$, and transforms it back into the original scalar variable $v$, i.e., executes $v=\hat{v}[\beta]$. The reason for this vectorization is to avoid clashes between the values that are accessed by the fragments while $\hat{b}[i]$ do $\hat{c}[i]$, for different values of $i$.

3. The last hypothesis we make is that the scalar variables initialized by the statement $c_{1}$ are not modified by $c$ or $c_{2}$ after vectorization. This is a reasonable assumption to make, since data structure accesses are in most cases confined to the inner loop. This allows us to ignore dependencies between these instructions and the rest of the loop.

As before, for every $i, j \in \mathbb{N}$ s.t. $i<j \leq \beta$ we need a function $\lambda_{i, j}: \mathbb{N} \rightarrow$ $\mathcal{S}$ mapping iterations to synchronization events. In this case, the parametric relation $\Omega_{i, j}$ takes into account the last instructions of the outer loop. We require, if $\left(n, \lambda_{i, j}(m)\right) \in \Omega_{i, j}$ and for every $\epsilon \subseteq \mathcal{S}$ and $\sigma \in \Sigma$, that:

$$
\llbracket \hat{c}[i]^{n} ; \hat{c}[j]^{m-1} ; \hat{c}[i]^{k} ; \hat{c_{2}}[i] ; \hat{c}[j] \rrbracket \equiv{ }_{(\sigma, \epsilon)} \llbracket \hat{c}[i]^{n} ; \hat{c}[j]^{m-1} ;\left(\hat{c}[i]^{k} ; \hat{c_{2}}[i] \| \hat{c}[j]\right) \rrbracket
$$


The transformation is similar to the one performed for sequences of loops. Since inner loops are syntactically equal, the value of induction variable $l$ corresponding to the outer loop is used to distinguish between different iterations. The transformation follows, thus, the scheme:

$$
\begin{aligned}
& \text { while } a \text { do } \quad \tau_{c, l-1} \rightarrow\left(\hat{c_{1}}[l] ; \tau_{c_{1}, l} !\right) ; \\
& \quad \text { while } \hat{b}[l] \text { do }\left(\bigcup_{1 \leq j<l} \lambda_{l, j}(h) \rightarrow\left(\hat{c}[l] ; \bigcup_{l<j \leq \beta} \omega_{l, j}(h) ! ; \hat{c_{2}}\left[h^{\prime}\right]\right)\right) ; \\
& \text { sync }
\end{aligned}
$$

Notice that the order in which the instances of $\hat{c}[l]$ are executed is preserved.

\subsection{Motivating Example Revisited}

Our motivating example, mergesort, was annotated with synchronization statements that follow the guidelines described in our transformation. If we take two consecutive iterations of the main loop of the program, we can sketch the constructs we have presented in our theoretical model.

Starting from the original code, we need first to vectorize the variables that parameterize our inner loop. In our example this is variable $i$. Since we need to spawn a new procedure in order to launch (possibly) a new thread, we encapsulate the inner loop in a function call, which receives $i$ as a parameter. Then, the stack allocation scheme automatically vectorizes variable $i$ for us, since now each iteration will possess its own copy of $i$, independent from the others, and initialized to the value which each iteration would see in a sequential execution. The only problem here consists in working with a language which allows function calls to be made to run in parallel. Later we will explain how we deal with this issue in practice.

In the original program, the variable $c$ is the expression used for writing in the array, and furthermore it is the lowest variable which is read or written in the array. On the other side, the variable $r$ is the highest variable which is read, this is a consequence of the initial state of the inner loop and is preserved in the loop body. We can analyze the loop and determine that $\mathrm{c}$ is monotonically increasing. It follows that if we have two consecutive iterations, $i$ and $i+1$, of the loop, the latter cannot proceed unless it can assure that the value of $\mathrm{c}^{\mathrm{i}}$ is bigger than that of $r^{i+1}$.

Thus, the following piece of code is added to the original code:

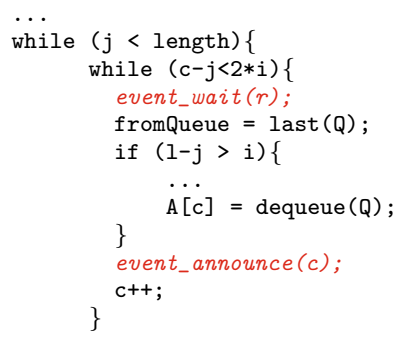

${ }^{1}$ We use superscripts to denote which loop variables belong to and subscripts to refer to the value of the variable at a given iteration of its loop. 


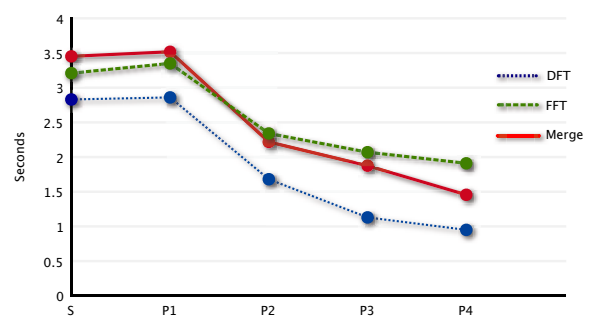

(a) Computation Time

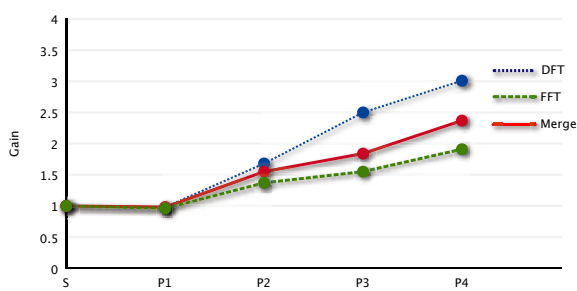

(b) Speedup

Fig. 4. Experimental Results

Our function $\lambda$ essentially maps $m \rightarrow r_{\mathrm{m}}$. It becomes apparent now that our $\Omega$ relation must relate every tuple $(n, \lambda(m))$ where $c_{n}^{i+1}$ is larger than $r_{m}^{i}$.

We now need to determine $\lambda$ and $\Omega$ for every other possible combination of iterations. But since the same loop is repeated, with the same properties, we require the same condition to advance, namely $\mathrm{r}_{\mathrm{m}}^{\mathrm{i}}<\mathrm{c}_{\mathrm{n}}^{\mathrm{j}}$, and thus $\Omega_{i, j}$ again contain pairs $\left(n, \lambda_{i, j}(m)\right)$ which meet that condition.

\section{Experimental Results}

We have experimented with the parallelizing transformation taking as input a program written in a subset of $C$ and returning a Cilk 5. program. Cilk is an extension of $C$ for multithreaded parallel programming, that provides a lightweight thread model based on job stealing.

We proceed by annotating the source program with Cilk statements for thread creation and synchronization, using Cilk locks and spawn procedures to implement event signaling and efficient variable synchronization. We encapsulate inner loops in spawned procedures, and use the $C$ stack allocation scheme to efficiently allocate memory for vectorization.

The proposed transformation has been applied to well-known algorithms that traverse arrays to obtain information as to the applicability and the efficiency of our approach. In all cases, the transformation yields good results unless the input size is tiny enough to make the synchronization overhead relatively significant.

For our tests we have used a 64bit Intel(R) Core(TM)2 Quad CPU at 2.4 $\mathrm{GHz}$ clock speed, 1GB of DIMM $800 \mathrm{MHz}$ memory, running GNU/Linux.

In all cases we have labeled the graphics with $\mathrm{S}$ for the sequential (unmodified) algorithm, running on a single processor, and we have labeled Pn for our modified, pipelined algorithm with $\mathrm{n}$ processors.

Figure 4 shows the computing time and the relative performance gain of the $\mathrm{DFT}^{2}$, FFT3 3 , and MergeSort algorithms run under the different conditions we have explained. The pipelined version of our DFT program is slightly slower while

\footnotetext{
${ }^{2}$ Discrete Fourier Transform.

${ }^{3}$ Fast Fourier Transform.
} 
running with only one processor, due to the overhead of synchronization variable allocation and signaling. Once we augment the number of available processors the amount of time spent computing starts to decrease as the several runs on the array on which we are working start to (safely) overlap. The efficiency gain is almost linear, but of course the overhead of signaling and also the thread creation and manipulation overhead add some extra work to the computation. The algorithm used is well suited for our transformation since it copies the input array and then modifies one element at a time incrementally, allowing several elements to be modified at the same time without interference.

Our experiments with an FFT algorithm also yield good results, though not as good as with the DFT algorithms. The reason for this is that unlike DFT, FFT traverses the input array heavily and performs the computation in-place, so it slowly gives up resources and thus the overlapping of different traversals is smaller. Nevertheless, some performance gain is indeed achieved in our pipelined version of the algorithm, roughly a $50 \%$ gain with 4 processors. The pipelined version is still outperformed by the sequential one in the case we have a single processor available, again due to synchronization overheads.

The last benchmark we present is that of our motivating example, namely mergesort. This algorithm also traverses an array several times incrementally, which allows us to obtain greater benefits from our transformation. The benchmarks were made sorting an array of one million elements. The results show that our transformation yields a $240 \%$ efficiency increase by overlapping the merging steps that are otherwise run sequentially, for a 4 processor machine.

\section{Related Work}

Ottoni et al. 13 proposed a technique called Decoupled Software Pipelining (DSWP) to extract the fine-grained parallelism hidden in most applications. The process is automatic, and general, since it considers non-scientific applications in which the loop iterations have heavy data dependencies. It provides a transformation that is slightly different to typical loop parallelization, in which each iteration is assigned alternately to each core, with an appropriate synchronization to prevent data races. As a result, no complete iteration is executed simultaneously with another one, since every iteration has a data dependence with every other one. Instead of alternating each complete loop iteration on each core, DSWP splits each loop body before distributing them among the available cores. This technique improves the locality of reference of standard parallelization techniques, and thus reduces the communication latency. It is effective in a more general set of loop bodies, but it does not take advantage of the eventual data independence hidden in scientific algorithms.

A recent experimental study [10] analyzes particular cases in which standard automatic parallelization fails to introduce significant improvements. This is the case of applications that manipulate complex and mutable data structures, such as Delauney mesh refinement and agglomerative clustering. The authors propose a practical framework, the Galois system, that relies on syntactic constructs to 
enable programmers to hint to the compiler on parallelization opportunities and an optimistic parallelization run-time to exploit them. Due to the unpredictability of irregular operations on mutable and complex data structures, the Galois framework is mostly based on runtime decisions and backtracking, and does not exploit statically inferred data dependence.

Data Parallel Haskell [14] (DPH) provides nested data parallelism to the existing functional language compiler GHC. Flat parallelism is restricted to the concurrent execution of sequential operations. Nested parallelism generalizes flat parallelism by considering the concurrent execution of functions that may be executed in parallel, and thus provides a more general and flexible approach, suitable for irregular problems. DPH extends Haskell with parallel primitives, such as parallel arrays and a set of parallel operations on arrays. The compiler compiles these parallel constructions by desugaring them into the GHC Core language, followed by a sequence of Core-to-Core transformations. DPH is a notable framework for the specification of concurrent programs, but the compiler is not intended to automatically discover parallel evaluations.

In a different line of work, the Manticore project is developing a parallel programming language for heterogeneous multi-core processor systems [3]. A main feature of the language is the support for both implicit and explicit threading. Nevertheless, as a design choice, it avoids implicit parallelism (i.e., it requires the programmer to hint parallelism by providing annotations) since they claim implicit parallelism to be only effective for dense regular parallel computations.

The goal of the Paraglide project at IBM is to assist the construction of highly-concurrent algorithms. The Paraglider tool [17] is a linearization-based framework to systematically construct complex algorithms manipulating concurrent data structures, from a sequential implementation. This approach combines manual guidance with automatic assistance, focusing mainly on fine-grained synchronization.

\section{Conclusion}

Synchronized pipelining is a parallelization technique that relies on eventual independence, a new refinement of the established notion of independence, to successfully transform programs with nested loops. This paper has set the theoretical foundations of the transformation, and showed its practical benefits on representative examples.

Future work includes applying this transformation to general recursive procedures, which is a possibility if the program is first transformed into an iterative version of itself. This is a widely studied optimization problem [1] which can significantly improve performance. Other lines of research include applying the transformation to languages that manipulate the heap. Many concepts developed in this paper are largely independent of the underlying programming language, and the main issue is rather to find an analysis to detect independence. Recent work on the use of shape analysis and separation logic for detecting data dependence and for parallelization provide a good starting point (e.g., [15[16 86 12] ). 


\section{References}

1. Allan, V., Jones, R., Lee, R., Allan, S.J.: Software pipelining. ACM Computing Surveys 27(3), 367-432 (1995)

2. Fahringer, T., Scholz, B.: A unified symbolic evaluation framework for parallelizing compilers. IEEE Transactions on Parallel and Distributed Systems PDS-11(11), 1105-1125 (2000)

3. Fluet, M., Rainey, M., Reppy, J., Shaw, A.: Implicitly-threaded parallelism in manticore. In: Hook, J., Thiemann, P. (eds.) ICFP, pp. 119-130. ACM, New York (2008)

4. Gerlek, M., Stoltz, E., Wolfe, M.: Beyond induction variables: detecting and classifying sequences using a demand-driven SSA form. ACM Transactions on Programming Languages and Systems 17(1), 85-122 (1995)

5. Supercomputing Technologies Group: Cilk 5.4.6 reference manual (1998)

6. Gulwani, S., Tiwari, A.: An abstract domain for analyzing heap-manipulating low-level software. In: Damm, W., Hermanns, H. (eds.) CAV 2007. LNCS, vol. 4590, pp. 379-392. Springer, Heidelberg (2007)

7. Hennessy, J., Patterson, D.: Computer Architecture: a quantitative approach. Morgan Kaufman, San Francisco (2003)

8. Hummel, J., Hendren, L., Nicolau, A.: A general data dependence test for dynamic, pointer-based data structures. In: PLDI, pp. 218-229 (1994)

9. Joyner, M., Budimlic, Z., Sarkar, V.: Optimizing array accesses in high productivity languages. In: Perrott, R.H., Chapman, B.M., Subhlok, J., de Mello, R.F., Yang, L.T. (eds.) HPCC 2007. LNCS, vol. 4782, pp. 432-445. Springer, Heidelberg (2007)

10. Kulkarni, M., Pingali, K., Walter, B., Ramanarayanan, G., Bala, K., Chew, L.P.: Optimistic parallelism requires abstractions. In: Ferrante, J., McKinley, K. (eds.) PLDI, pp. 211-222. ACM, New York (2007)

11. Liu, Y., Stoller, S.: From recursion to iteration: What are the optimizations? In: PEPM, pp. 73-82 (2000)

12. Marron, M., Kapur, D., Stefanovic, D., Hermenegildo, M.: Identification of HeapCarried Data Dependence Via Explicit Store Heap Models. In: Amaral, J.N. (ed.) LCPC 2008. LNCS, vol. 5335, pp. 94-108. Springer, Heidelberg (2008)

13. Ottoni, G., Rangan, R., Stoler, A., August, D.I.: Automatic thread extraction with decoupled software pipelining. In: MICRO, pp. 105-118. IEEE Computer Society, Los Alamitos (2005)

14. Peyton Jones, S.: Harnessing the multicores: Nested data parallelism in haskell. In: Ramalingam, G. (ed.) APLAS 2008. LNCS, vol. 5356, p. 138. Springer, Heidelberg (2008)

15. Raza, M., Calcagno, C., Gardner, P.: Automatic parallelization with separation logic. In: Castagna, G. (ed.) ESOP 2009. LNCS, vol. 5502, pp. 348-362. Springer, Heidelberg (2009)

16. Rugina, R., Rinard, M.: Automatic parallelization of divide and conquer algorithms. In: PPOPP, pp. 72-83 (1999)

17. Vechev, M., Yahav, E.: Deriving linearizable fine-grained concurrent objects. In: Gupta, R., Amarasinghe, S. (eds.) PLDI, pp. 125-135. ACM, New York (2008) 